\title{
MEASUREMENT OF THE VACUUM DECAY RATE OF ORTHOPOSITRONIUM FORMED IN AN MgO-LINED CAVITY
}

\author{
D.W. GIDLEY \\ Department of Physics, University of Michigan, Ann Arbor, MI 48109, USA \\ and \\ P.W. ZITZEWITZ \\ Department of Natural Sciences, University of Michigan-Dearborn, Dearborn, MI 48128, USA
}

Received 16 August 1978

\begin{abstract}
Orthopositronium decay rates are measured in $\mathrm{MgO}$-lined cavities with various volumes and entrance apertures. Systematic effects of the entrance aperture, cavity geometry, and collisional pick-off are measured. The vacuum decay rate is determined to be $7.050 \pm 0.013 \mu \mathrm{s}^{-1}$.
\end{abstract}

We report the results of our continued investigation of the annihilation decay rate, $\lambda$, of orthopositronium (o-Ps) $\left(1^{3} \mathrm{~S}_{1}\right)$ formed in an evacuated $\mathrm{MgO}$-lined cavity. Our first measurement [1] in 1976 using this method yielded a value of $7.09 \pm 0.02 \mu \mathrm{s}^{-1}$. Since then a new theoretical value [2] of $7.0386 \pm 0.00016$ has been calculated and we have measured $\lambda=7.056$ $\pm 0.007 \mu \mathrm{s}^{-1}$ in isobutane gas by extrapolation to zero gas density [3]. In a recent letter, Canter et al. [4] have reported that the decay rate of o-Ps formed in a $\mathrm{MgO}$-lined rf cavity is $7.122 \pm 0.012 \mu \mathrm{s}^{-1}$ and they suggest that the high decay rates measured using this method may be due to pick-off quenching of Ps during wall collisions. However, our new result for $\lambda$ is significantly lower than these two previous measurements. It is in agreement with both the experimental value measured in gases and the theoretical value. We will discuss the systematic errors associated with interpreting this result as the vacuum decay rate and show why our earlier result is too high.

The interaction region is shown in fig. 1 (also see ref. [1]). A beam of slow positrons $(\approx 400 \mathrm{eV})$ are electrostatically focused onto the MgO-coated cone of a channel electron multiplier (CEM), where positronium is formed with about $15 \%$ efficiency. Secondary electrons, expelled by the incident positron, are de-

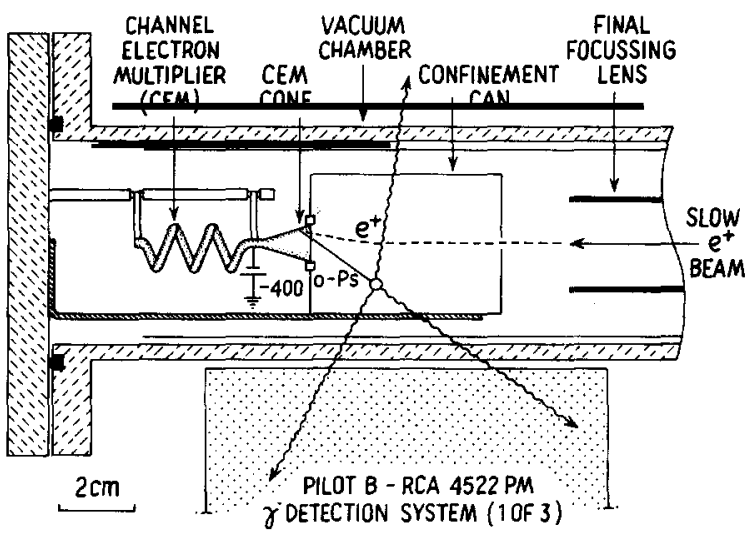

Fig. 1. Evacuated interaction region.

tected by the CEM and serve as the "start" signal. o-Ps is confined to a region of nearly uniform $\gamma$ ray detection efficiency by an MgO-coated cavity and the annihilation $\gamma$ rays ("stop" signal) are detected by 3 scintillation detectors surrounding the interaction region. More details of the apparatus and the timing system can be found in ref. [1].

Since the earlier experiment a number of improvements have been made. The time-to-amplitude convert- 
er has been replaced with a Hewlett Packard 5345A time digitizer interfaced with the MCA memory. Timing accuracy is better than $1 \mathrm{ppm}$ and tests at the $0.1 \%$ level have not revealed any non-linearity. The positron beam strength has been increased by a factor of three to $1500 \mathrm{~s}^{-1}$ and focusing improved so that the cavity entrance hole diameter can be as small as $2 \mathrm{~mm}$. Replacement of the $10 \mathrm{~cm} \times 10 \mathrm{~cm} \mathrm{NaI} \gamma$ detectors with Pilot B scintillator and elimination of the prompt rejection system have allowed us to reduce the timing electronics to a very simple system: an amplifier and a leading-edge discriminator for each of the 4 detectors, a stop signal OR gate, and the digitizer-MCA system.

Each lifetime spectrum of typically 30 million o-Ps events was fitted by a three-parameter maximum-likelihood program [5] to

$N(t)=A \mathrm{e}^{-\lambda_{0} t}+B$,

where $\lambda_{0}$ is the decay rate of o-Ps in the cavity and $B$ is the intensity of uncorrelated background events. Typically $A / B=500$ at $80 \mathrm{~ns}$ beyond the prompt peak. The fitted decay rate is observed to decrease by about $0.4 \%$ and asymptotically approach a constant value as the starting point of the fitting program is successively stepped out in $48 \mathrm{~ns}$ intervals from $t=76 \mathrm{~ns}$ to $t=700$ ns. Consistent with ref. [4] the decay rate is a constant beyond $t=300 \mathrm{~ns}$ and we interpret this asy mptotic value to be the o-Ps decay rate in the cavity. In our previous experiment the asymptotic approach was obscured by an apparent systematic oscillation in the fitted decay rate of $0.015 \mu \mathrm{s}^{-1}$. The decay rate at $t$ $=300 \mathrm{~ns}$ was about $0.015 \mu \mathrm{s}^{-1}$ below the value quoted in ref. [1]. A second correction to this earlier work will be discussed later.

If o-Ps is free in the evacuated cavity with an average wall collision rate, $\nu$, the observed decay rate, $\lambda_{0}$, should be

$\lambda_{0}=\lambda+P_{\mathrm{a}} \nu+c \nu A / S$,

where $P_{\mathrm{a}}$ is the probability of annihilation per wall collision, $S$ is the surface area of the cavity, $A$ is the area of the entrance hole and $c$ is the probability that Ps escaping out the entrance hole will not be detected. The second term is the collisional pick-off rate and the last term is the "disappearance" rate of Ps through the entrance hole. If Ps bounces isotropically from a wall the collision rate is proportional to the surface-to- volume ratio of the cavity and the average velocity, $\bar{v}$, $\nu=(\bar{v} / 4)(S / V)$.

Eq. (2) becomes

$\lambda_{0}=\lambda+P_{\mathrm{a}}(\bar{v} / 4)(S / V)+c(\bar{v} / 4)(A / V)$.

The decay rates observed using 3 different cavity volumes $\left(13 \mathrm{~cm}^{3}, 29 \mathrm{~cm}^{3}\right.$, and $\left.58 \mathrm{~cm}^{3}\right)$ with different entrance hole areas are plotted versus $A / V$ in fig. 2. The fact that all the data lie on the same straight line indicates that the dominant effect is due to the entrance hole. Since the surface-to-volume ratio $(S / V)$ for the smallest cavity is 1.7 times higher than the largest cavity and the data are within one standard deviation $\left(0.005 \mu \mathrm{s}^{-1}\right)$ we estimate the pick-off term to be less than $0.1 \%$. For $1 \mathrm{eV}$ Ps this corresponds to $P_{\mathrm{a}}$ $<3 \times 10^{-4}$ compared to $P_{\mathrm{a}} \approx 10^{-6}$ in bulk $\mathrm{MgO}$ powder. In addition, the slope of the line in fig. 2 can be related to the average velocity using eq. (4) once $c$ is known. We find that $\bar{v}$ corresponds to an energy of approximately $0.7 \mathrm{eV}$, in good agreement with previous estimates of about $1 \mathrm{eV}[1,6]$.

From fig. 2 it can be seen why the 1976 data are too high. The relationship between cavity volume and entrance hole effect was not understood. Only the 58 $\mathrm{cm}^{3}$ cavity was used to estimate the effect of the entrance hole to be $0.01 \mu \mathrm{s}^{-1}$ at $A / V=5.5 \times 10^{-3}$ $\mathrm{cm}^{-1}$. This correction should have been $0.016 \mu \mathrm{s}^{-1}$ for the large cavity and $0.032 \mu \mathrm{s}^{-1}$ for the two runs

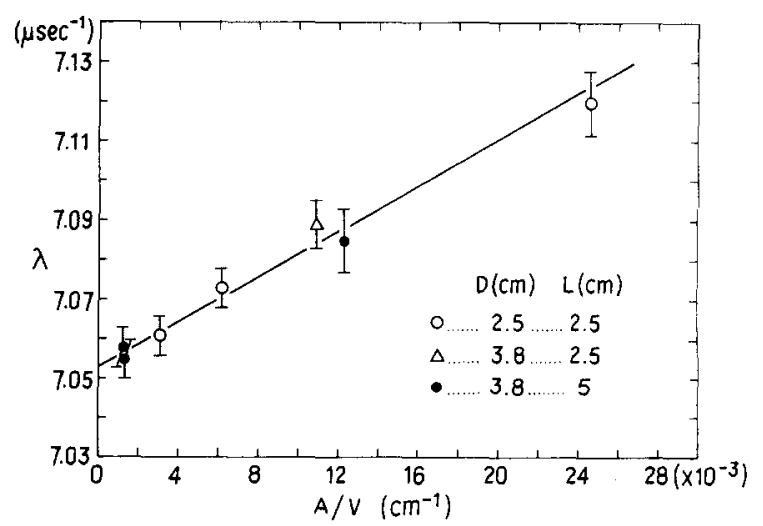

Fig. 2. Plot of decay rate versus the entrance hole area-to-cavity volume ratio for three different cavities. Chi-square deviation of weighted least-square linear fit is 1.3 for 5 degrees of freedom. The probability for a chi-square so low is only $7 \%$ and indicates that using the error estimate for the fitted decay rate at $t=300 \mathrm{~ns}$ is conservative. 
with the shorter cavity. With this correction and using the fitted decay rate at $t=300 \mathrm{~ns}$ the average of the three 1976 runs is $7.053 \pm 0.008 \mu \mathrm{s}^{-1}$.

The data in fig. 2 are subject to a small volume-dependent systematic shift in the horizontal axis due to a small annular opening of $0.1 \mathrm{~mm}$ between the CEM cone and the cavity wall. The resultant increase in the Ps disappearance rate, assumed to be proportional to $A / V$, was determined by using an enlarged opening of $1.0 \mathrm{~mm}$. The intercepts in fig. 2 for the large and small cavities should be decreased by $0.001 \mu \mathrm{s}^{-1}$ and 0.004 $\mu \mathrm{s}^{-1}$ yielding $7.052 \pm 0.004 \mu \mathrm{s}^{-1}$ and $7.050 \pm 0.005$ $\mu \mathrm{s}^{-1}$, respectively.

Fig. 3 shows the results of using the $29 \mathrm{~cm}^{3}$ cavity with the CEM cone sealed to the cavity wall. The intercept is $7.049 \pm 0.004 \mu \mathrm{s}^{-1}$. Averaging the intercepts for each of the 3 cavity volumes yields $7.050 \pm 0.0025$ $\mu \mathrm{s}^{-1}$. This result is in agreement with the corrected 1976 data. The decay rate obtained by Canter et al., $7.122 \pm 0.012 \mu \mathrm{s}^{-1}$, even when corrected for an estimated $0.025 \mu \mathrm{s}^{-1}$ entrance hole effect is still significantly higher than our present value.

In order to determine the vacuum decay rate of o-Ps we have shown that the major correction to the observed decay rates is associated with the cavity entrance hole and that it is well described by eq. (4). The systematic error in making this extrapolation to $A / V$ $=0$ is negligible. By using cavities with $S / V$ between $1.4 \mathrm{~cm}^{-1}$ and $2.4 \mathrm{~cm}^{-1}$ we have shown that the collisional pick-off term in eq. (4) is consistent with zero at the level of $0.1 \%$. The most significant remaining systematic effect is due to the diffusion of o-Ps into

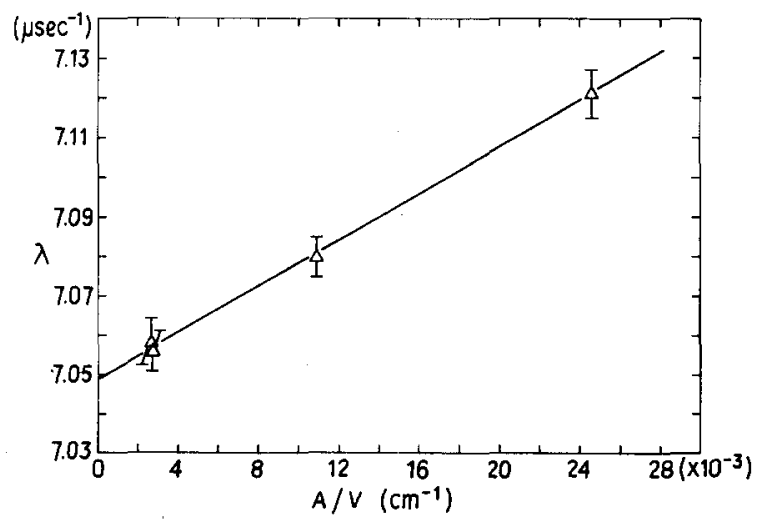

Fig. 3. Plot of $\lambda$ versus $A / V$ for a $3.8 \mathrm{~cm} \times 2.5 \mathrm{~cm}$ cavity. Chisquare is only 0.1 for 2 degrees of freedom (see fig. 2 caption). the $\mathrm{MgO}$ layer on the CEM cone where the decay rate is about $3 \%$ higher [1] than the vacuum value. Since the coating thickness of $0.05-0.1 \mathrm{~mm}$ is estimated to be at least one diffusion length for $1 \mathrm{eV}$ Ps and all Ps is formed on or in the cone layer some fraction of the o-Ps will decay in the powder. In ref. [1] we showed that after $t=35 \mathrm{~ns}$ no more than $10 \%$ of all o-Ps annihilations occurred in the vicinity of the cone. At longer times this fraction should decrease as Ps escapes from the cone. The $0.4 \%$ drop in the fitted decay rate between $t=76 \mathrm{~ns}$ and $t=300 \mathrm{~ns}$ may be a manifestation of the escape of Ps from the MgO coating. This effect is further complicated by the nonuniform $\gamma$-ray detection efficiency since a systematically lower fitted decay rate may result from the time-delayed escape of Ps from a region of lower to higher detection efficiency. However, if $5 \%$ of the o-Ps is inescapably trapped on the MgO-coated cone with a $3 \%$ pick-off rate the observed decay rate will be $0.15 \%$ higher than the vacuum value. Such an error cannot be ruled out at this time since the effect would be independent of the cavity dimensions and show no decrease in the fitted decay rate as the start channel is stepped out.

Adding the possible systematic errors in quadrature yields, as a measurement of the vacuum decay rate of o-Ps, $\lambda=7.050 \pm 0.013 \mu \mathrm{s}^{-1}$. We note that if such systematic errors exist it is more likely that correction for such errors will result in a lower decay rate.

We thank Professor A. Rich for continuing participation and interest in this experiment. We also thank G.W. Ford, J.R. Freeling, and E. Sweetman for helpful discussions. This work was supported by the National Science Foundation under Grant No. PHY77-28139; the Research Corporation; the Rackham Graduate School, the Institute of Science and Technology, and the Office of the Vice-President for Research of The University of Michigan.

[1] D.W. Gidley, P.W. Zitzewitz, K.A. Marko and A. Rich, Phys. Rev. Lett. 37 (1976) 729.

[2] W.E. Caswell, G.P. Lepage and J. Sapirstein, Phys. Rev. Lett. 38 (1977) 488;

W.E. Caswell and G.P. Lepage, private communication.

[3] D.W. Gidley, A. Rich, P.W. Zitzewitz and D.A.L. Paul, Phys. Rev. Lett. 40 (1978) 737.

[4] K.F. Canter, B.O. Clark and I.J. Rosenberg, Phys. Lett. 65A (1978) 301.

[5] P.H.R. Orth, W.R. Falk and G. Jones, Nucl. Instr. Meth. 65 (1968) 301.

[6] A.P. Mills Jr., K.F. Canter and S. Berko, Phys. Rev. Lett. 34 (1975) 1541. 